\title{
Controllability for one-dimensional nonlinear wave equations with degenerate damping *
}

\author{
Jieqiong $\mathbf{W u},{ }^{\dagger}$ Xianzheng Zhu, Shugen Chai \\ School of Mathematical Sciences, Shanxi University, \\ Taiyuan, Shanxi 030006, China
}

Abstract Internal exact controllability for the nonlinear wave equation in one space dimension

$$
y_{t t}-y_{x x}+g(y) y_{t}=h
$$

is studied, where $g(\cdot)$ is a nonnegative function. For the case, $\limsup _{|s| \rightarrow \infty} \frac{g(s)}{\ln |s|}<\gamma$, we obtain the global exact controllability for the equation with Dirichlet boundary condition. The proof is based on the combination of fixed-point arguments and explicit observability estimates for the linearized wave equation with a potential that depends on both $x$ and $t$. For the case, $g(s)=|s|$, we only get a local exact controllability by means of Banach fixed-point theorem.

Keywords exact controllability; wave equations; degenerate damping

\section{Introduction}

Let $\Omega=(0,1)$ and $T>0$. Set $H=H_{0}^{1}(\Omega) \times L^{2}(\Omega)$ throughout this paper. Let $\omega=(\alpha, \beta)$ be a subinterval of $\Omega$ with $0 \leq \alpha<\beta \leq 1$. Denote by $1_{\omega}$ the characteristic function of $\omega$. This paper is concerned with the exact controllability of the nonlinear wave equation in one space dimension

$$
\left\{\begin{array}{l}
y_{t t}-y_{x x}+g(y) y_{t}=h 1_{\omega}, \quad \text { in }(0, T) \times \Omega \\
y(t, 0)=y(t, 1)=0, \quad \text { in }(0, T) \\
y(0, x)=y^{0}(x), \quad y_{t}(0, x)=y^{1}(x), \quad \text { in } \Omega,
\end{array}\right.
$$

where $h \in L^{2}(\omega \times(0, T))$ is the control, $\left(y^{0}, y^{1}\right) \in H$ and $g(\cdot): R \rightarrow[0, \infty)$ is a nonnegative continuous function satisfying $g(0)=0$.

The exact controllability problem of (1.1) may be formulated as follows: for any $\left(y^{0}, y^{1}\right),\left(z^{0}, z^{1}\right) \in$ $H$, is it possible to find a control $h \in L^{2}(\omega \times(0, T))$ such that the solution of (1.1) satisfies

$$
\left(y(T, x), y_{t}(T, x)\right)=\left(z^{0}(x), z^{1}(x)\right), \quad x \in \Omega ?
$$

The wave equation in (1.1) is a special case of

$$
y_{t t}-\Delta y+\mathcal{R}\left(t, x, y, y_{t}\right)=\mathcal{F}(x, y)+h 1_{\omega},
$$

${ }^{*}$ This work is supported by the National Science Foundation of China for the Youth(61104129).

${ }^{\dagger}$ Corresponding author, e-mail: jieqiong@sxu.edu.cn

(C) 2017. This manuscript version is made available under the Elsevier user license http://www.elsevier.com/open-access/userlicense/1.0/ 
where $\mathcal{R}$ and $\mathcal{F}$ satisfy the structural conditions: $z \mathcal{R}(t, x, y, z) \geq 0, \mathcal{R}(t, x, y, 0)=\mathcal{F}(x, 0)=0$ and $\mathcal{F}(x, y) \sim|y|^{p-1} y$ for large $y$. Many examples of (1.2) arise in quantum field theory and some important mechanical applications, see [14] and [20].

The existence of solutions for various special cases of (1.2) with $h \equiv 0$ (which is a pure PDE problem) has been widely studied in literature, see for example [19] and [4]. In particular, if $\mathcal{F}(x, y) \equiv$ 0 , then damping terms of various forms are known to yield existence of global solutions (cf. $[1,3,8,12])$. Thus (1.1) has a solution $y \in C\left([0, T] ; H_{0}^{1}\right) \cap C^{1}\left([0, T] ; L^{2}(\Omega)\right)$. The uniqueness of the solution of (1.1) is easy to prove. We remark that the damping term $g(y) y_{t}$ was called degenerate in literature, see for example [4].

There are also some results on the exact controllability problem for equation (1.2). When $\mathcal{F}(x, y) \equiv$ 0 and $\mathcal{R}\left(t, x, y, y_{t}\right)=f(y)$ satisfying

$$
\lim _{|s| \rightarrow \infty} \frac{f(s)}{|s| \ln ^{r}|s|}=0
$$

Zuazua [30] proved, for $r=2,(1.2)$ with Dirichlet boundary condition in one space dimension was globally exactly controllable in the natural energy space $H$. The proof was based on HUM (Hilbert uniqueness method) and Leray-Schauder's fixed-point theorem. For $r>2$, it was also proved in [30] the system was not exactly controllable in any time $T>0$ (the solution blew up). However, to our best knowledge, the exact controllability result of Zuazua [30] is not yet to be extended to higher dimensions. Later on, the authors of [11] and [9] obtained the controllability results for multidimensional semilinear wave equations, but for the case: $0 \leq r<\frac{3}{2}$ in (1.3). The author of [22] studied the exact controllability of two hyperbolic equations (one semilinear, and the other one linear) coupled in cascade through the boundary where the assumption on the nonlinearity $f(y)$ was the same as that in [11] and [9] .

When $\mathcal{R}\left(t, x, y, y_{t}\right)$ satisfies in a neighbourhood of $\left(y, y_{t}\right)=(0,0)$ that

$$
\mathcal{R}\left(t, x, y, y_{t}\right)=O\left(|y|^{2}+\left|y_{t}\right|^{2}\right)
$$

the authors of [28] proved that the equation (1.2) with various type boundary conditions is locally exactly controllable in $H^{s+1}(\Omega) \times H^{s}(\Omega), s \geq 2$, see also [27]. We refer the readers to [5, 17, 21, 23, 24,6] for more results of controllability for damped hyperbolic equations.

The local exact boundary controllability was also established for quasi-linear wave equations in several space dimensions by using the constructive method in [28]. More results on quasilinear cases can be found in [25] and [29]. The author of [25] introduced some latest advances on control of the quasilinear wave equation in higher dimensions by combining the Riemannian geometrical approach with some methods from nonlinear partial differential equations where the quasilinearity arises in the principal part of the systems. The authors of [29] studied the local exact controllability using an extension method.

We remark that the equation (1.1) with degenerate damping terms is quite different from those types of equations mentioned above. In this paper we are devoted to the analysis of the controllability 
of (1.1). To our knowledge, there are no previous controllability results for this kind of equation. Our main results read as follows.

Theorem 1.1. Set $T_{0}=2 \max \{\alpha, 1-\beta\}, T>T_{0}$. Assume that

$$
\limsup _{|s| \rightarrow \infty} \frac{g(s)}{\ln |s|}<\gamma .
$$

Then there exists $\gamma>0$ such that if (1.4) holds then system (1.1) is exactly controllable in time T.

Theorem 1.2 Let $T>T_{0}$. Then there exists $r_{0}>0$ such that for every $\left(y^{0}, y^{1}\right),\left(z^{0}, z^{1}\right) \in H$ with $\left\|\left(y^{0}, y^{1}\right)\right\|_{H} \leq r_{0}$ and $\left\|\left(z^{0}, z^{1}\right)\right\|_{H} \leq r_{0}$, there exist

$$
y \in B:=C\left([0, T] ; H_{0}^{1}(\Omega)\right) \cap C^{1}\left([0, T] ; L^{2}(\Omega)\right)
$$

and $h \in L^{2}((0, T) \times \omega)$ satisfying (1.1) with $g(s)=|s|$ such that

$$
y(T, x)=z^{0}(x), \quad y_{t}(T, x)=z^{1}(x), \quad x \in \Omega .
$$

The proof of Theorem 1.1 is based on the combination of Schaefer's fixed-point arguments and explicit observability estimates of the linearized wave equation with a potential that depends on both $x$ and $t$. For such an explicit observability estimate, we refer the reader to [26] and [9]. The authors of [26] and [9] used Carleman estimates and usual energy estimates to obtain the explicit observability inequality for linear wave equations with lower order terms. We note that when the author of [26] considered the corresponding controllability problem, the coefficient of the damping term was assumed to be once differentiable (see Theorem 4.1 of [26]). That was due to the Hilbert uniqueness method (HUM) used in that paper which led the term $\left(p_{2} \varphi\right)_{t}$ (see (4.3) of [26]) to be involved inevitably in the dual system. But when we consider the controllability of the linearized system in the present paper, the coefficient of the damping term does not satisfy the $C^{1}$-regularity. Consequently, we can not directly follow the approach of [26] and [9] to prove the exact controllability of the linearized system. In fact, the method that we use here is rather different from [26] and [9] and it is due to a classical result of functional analysis (see Lemma 2.2). The same method has been used in [7] to prove exact controllability for a linear wave equation with potentials. By this method we deduce a specific dual system which consists of two equations (see (2.11)) and avoid the high regularity requirement for coefficients of the damping term.

When $g$ satisfies the sublinear growth condition (1.4), we obtain global exact controllability for system (1.1) in Theorem 1.1. Otherwise, for the case $g(s)=|s|$, since the uniform boundedness of $y$ like (2.37) obtained in the proof of Theorem 1.1 is not available unless the initial and final states are both taken small enough, we only get local exact controllability as stated in Theorem 1.2. Instead of the Schaefer's fixed-point theorem applied in the proof of Theorem 1.1, we prove Theorem 1.2 by using the Banach fixed-point theorem. However, one could still expect the global exact controllability holds in the latter case owing to the global existence of the solution to (1.1). Unfortunately, we do not know yet whether that is true and how to verify that. 
The remainder of this paper is organized as follows. Section 2 is devoted to the proof of Theorem 1.1. The exact controllability for the nonlinear system is reduced to the exact controllability for the linearized system and an application of the fixed-point method, which will be done in Subsection 2.1 and 2.3, respectively. Subsection 2.2 is devoted to the obtention of an explicit observability inequality. We prove Theorem 1.2 in detail in Section 3.

\section{Proof of Theorem 1.1}

This section deals with the global exact controllability for the case of $g$ satisfying the assumption (1.4). We will introduce a linearized system for (1.1) and prove its exact controllability. Once the linearized system is controllable, by a fixed-point technique, we will prove the controllability for the nonlinear system.

Throughout this section, $T$ is fixed as $T>T_{0}$, and $C$ denotes a generic positive constant depending merely on $T$ and $\Omega$.

\subsection{The linearized system}

Given any $\left(y^{0}, y^{1}\right),\left(z^{0}, z^{1}\right) \in H$ and $a \in L^{\infty}((0, T) \times \Omega)$, introduce the linearized system for (1.1)

$$
\left\{\begin{array}{l}
z_{t t}-z_{x x}+g(a) z_{t}=h 1_{\omega}, \quad \text { in }(0, T) \times \Omega \\
z(t, 0)=z(t, 1)=0, \quad \text { in }(0, T) \\
z(0, x)=y^{0}(x), z_{t}(0, x)=y^{1}(x), \quad \text { in } \Omega .
\end{array}\right.
$$

We will prove the exact controllability of $(2.1)$ : for any $\left(y^{0}, y^{1}\right),\left(z^{0}, z^{1}\right) \in H$, there exists a control $h \in L^{2}((0, T) \times \omega)$ such that the solution of (2.1) satisfies

$$
z(T, x)=z^{0}(x), \quad z_{t}(T, x)=z^{1}(x), \quad x \in \Omega .
$$

Let us consider the following system

$$
\left\{\begin{array}{l}
\tilde{y}_{t t}-\tilde{y}_{x x}+g(a) \tilde{y}_{t}=h 1_{\omega} \quad \text { in }(0, T) \times \Omega \\
\tilde{y}(t, 0)=\tilde{y}(t, 1)=0 \quad \text { in }(0, T),
\end{array}\right.
$$

with the initial data

$$
\tilde{y}(0, x)=\tilde{y}_{t}(0, x)=0, \quad \text { in } \Omega .
$$

It follows from lemma 2.3 of [26] that for any $h \in L^{2}((0, T) \times \omega)$, problem $(2.3)$ with the initial data $(0,0)$ admits a unique solution $\tilde{y} \in C\left([0, T] ; H_{0}^{1}\right) \cap C^{1}\left([0, T] ; L^{2}(\Omega)\right)$, which satisfies

$$
\tilde{E}(t) \leq C e^{C\|g(a)\|_{\infty}} \int_{0}^{T} \int_{\omega} h^{2} d x d t, \quad \forall t \in[0, T]
$$


for some constant $C>0$, where $\|g(a)\|_{\infty}=\|g(a)\|_{L^{\infty}((0, T) \times \Omega)}$ and

$$
\tilde{E}(t)=\frac{1}{2} \int_{\Omega}\left(\tilde{y}_{t}^{2}(t, x)+\tilde{y}_{x}^{2}(t, x)\right) d x
$$

Given any $\left(y^{0}, y^{1}\right) \in H$, let $\bar{y}$ solve

$$
\left\{\begin{array}{l}
\bar{y}_{t t}-\bar{y}_{x x}+g(a) \bar{y}_{t}=0 \quad \text { in }(0, T) \times \Omega \\
\bar{y}(t, 0)=\bar{y}(t, 1)=0 \quad \text { in }(0, T) \\
\bar{y}(0, x)=y^{0}(x), \quad \bar{y}_{t}(0, x)=y^{1}(x), \quad \text { in } \Omega .
\end{array}\right.
$$

Let us assume that there exists a control $h \in L^{2}((0, T) \times \omega)$ such that the solution of (2.3) satisfies

$$
\tilde{y}(T, x)=z^{0}(x)-\bar{y}(T, x), \quad \tilde{y}_{t}(T, x)=z^{1}(x)-\bar{y}_{t}(T, x), \quad x \in \Omega .
$$

Then we have $\tilde{y}+\bar{y}$ solves (2.1) and satisfies (2.2). Thus the exact controllability of (2.1) is equivalent to the exact controllability of $(2.3)$.

In order to prove the exact controllability of (2.3), let us define a linear map

$$
F: L^{2}((0, T) \times \omega) \rightarrow H
$$

in the following way. Let $h 1_{\omega} \in L^{2}((0, T) \times \omega)$. Let $\tilde{y}$ be the corresponding solution of (2.3). Then $F\left(h 1_{\omega}\right)=\left(\tilde{y}(T, \cdot), \tilde{y}_{t}(T, \cdot)\right)$. It is easy to see from $(2.4)$ that $F$ is continuous.

One has the following lemma by the definition of $F$.

Lemma 2.1 The system (2.3) is exactly controllable if and only if $F$ is surjective.

We prove $F$ is surjective by using the following well-known lemma in functional analysis (see Proposition 2.16 in $[7])$.

Lemma 2.2 Let $H_{1}$ and $H_{2}$ be two Hilbert spaces. Let $F$ be a linear continuous map from $H_{1}$ into $H_{2}$. Then $F$ is surjective if and only if there exists $c^{*}>0$ such that

$$
\left\|x_{2}\right\|_{H_{2}} \leq c^{*}\left\|F^{*}\left(x_{2}\right)\right\|_{H_{1}} .
$$

Moreover, if (2.8) holds for some $c^{*}>0$, there exists a linear continuous map $G$ from $H_{2}$ into $H_{1}$ such that

$$
\begin{gathered}
F \circ G\left(x_{2}\right)=x_{2}, \quad \forall x_{2} \in H_{2}, \\
\left\|G\left(x_{2}\right)\right\|_{H_{1}} \leq c^{*}\left\|x_{2}\right\|_{H_{2}}, \quad \forall x_{2} \in H_{2} .
\end{gathered}
$$

In order to compute the adjoint operator of $F$, we borrow some ideas from [7]. Denote by $\mathcal{M}^{t r}$ the transpose of the matrix $\mathcal{M}$. Consider the system

$$
\begin{cases}P_{t}=Q, & \text { in }(0, T) \times \Omega \\ Q_{t}=P_{x x}+g(a) Q, & \text { in }(0, T) \times \Omega \\ P(t, 0)=P(t, 1)=0, & t \in(0, T), \\ P(T, x)=P^{T}(x), & Q(T, x)=Q^{T}(x), \quad \text { in } \Omega\end{cases}
$$


where $\left(P^{T}, Q^{T}\right)^{t r} \in H$.

Define an operator $A: D(A) \subset H \rightarrow H$ as follows.

$$
A=\left(\begin{array}{cc}
0 & I \\
\frac{\partial^{2}}{\partial x^{2}} & 0
\end{array}\right), \quad D(A)=\left(H^{2}(\Omega) \cap H_{0}^{1}(\Omega)\right) \times H_{0}^{1}(\Omega) .
$$

It's easy to check that $A^{*}=-A$. Denote $u=(P, Q)^{t r}, f(t, u)=(0, g(a) Q)^{t r}$ and $u^{T}=\left(P^{T}, Q^{T}\right)^{t r}$. Then (2.11) can be rewritten as the following abstract Cauchy problem:

$$
\left\{\begin{array}{l}
\frac{d}{d t} u(t)=A u(t)+f(t, u(t)), \quad t \in(0, T), \\
u(T)=u^{T} \in H
\end{array}\right.
$$

where $f$ is uniformly Lipschitz in $u \in H$. Therefore, for every $u^{T} \in H,(2.12)$ has a unique solution $u \in C([0, T] ; H)$ (see Chapter 6 of [18]).

Now we make $F^{*}$ explicit by the following lemma.

Lemma 2.3 Let $u^{T}=\left(P^{T}, Q^{T}\right)^{t r} \in H$. Then

$$
F^{*} u^{T}=Q 1_{\omega}
$$

where $u=(P, Q)^{t r} \in C([0, T] ; H)$ is the solution of $(2.12)$ with $u(T)=u^{T}$.

Proof By using the density argument we just need to prove (2.13) for classical solutions such that the following computations make sense. Noting the definition of $F$, from (2.3) and (2.12), integrating by parts, we get

$$
\begin{aligned}
& \left(u^{T}, F\left(h 1_{\omega}\right)\right)_{H} \\
= & \int_{0}^{1} P_{x}(T, x) y_{x}(T, x)+Q(T, x) y_{t}(T, x) d x \\
= & \int_{0}^{T} \int_{0}^{1}\left(P_{x} y_{x}+Q y_{t}\right)_{t} d x d t \\
= & \int_{0}^{T} \int_{0}^{1} P_{t x} y_{x}+P_{x} y_{t x}+Q_{t} y_{t}+Q y_{t t} d x d t \\
= & \int_{0}^{T} \int_{0}^{1}-P_{t} y_{x x}-P_{x x} y_{t}+Q_{t} y_{t}+Q\left(y_{x x}-g(a) y_{t}+h 1_{\omega}\right) d x d t \\
= & \int_{0}^{T} \int_{0}^{1}\left(Q-P_{t}\right) y_{x x}+\left(Q-P_{x x}-g(a) Q\right) y_{t}+Q h 1_{\omega} d x d t \\
= & \int_{0}^{T} \int_{\omega} Q h d x d t,
\end{aligned}
$$

which implies (2.13).

Thanks to Lemma 2.2, to show that $F$ is surjective, it is enough to prove the following theorem. Theorem 2.1 Let $T>T_{0}$. Then there exist $C_{1}>0$ and $C_{2}>0$ which are constants only depending on $T$ and $\Omega$ such that

$$
\left\|\left(P^{T}, Q^{T}\right)^{t r}\right\|_{H} \leq C_{1} e^{C_{2}\|g(a)\|_{\infty}}\|Q\|_{L^{2}((0, T) \times \omega)},
$$


for all $\left(P^{T}, Q^{T}\right)^{t r} \in H$.

We will prove Theorem 2.1 in Subsection 2.2.

From Lemma 2.1, Lemma 2.2 and Theorem 2.1, we arrive at the exact controllability of system $(2.3)$.

\subsection{The observability inequality}

The aim of this subsection is to prove Theorem 2.1. We begin with the following lemma and for the reader's convenience we give the proof of it as well. We remark that the technique of the proof originally comes from Haraux [13] and is similar to the proof of Lemma 3.7 of [23].

Lemma 2.4 Set $T_{0}=2 \max \{\alpha, 1-\beta\}, T>T_{0}$. Let

$$
f(t, x)=P_{t}^{2}(t, x)+P_{x}^{2}(t, x), \quad \forall(t, x) \in[0, T] \times \Omega .
$$

Then for all $T>T_{0}$, there exist $\delta>0, \eta>0$ and $C>0$ such that

$$
\begin{aligned}
& \int_{\frac{T}{2}-\delta}^{\frac{T}{2}+\delta} \int_{\beta-\eta}^{1} f(x, t) d x d t \leq C \int_{0}^{T} \int_{\alpha}^{\beta} f(x, t) d x d t, \\
& \int_{\frac{T}{2}-\delta}^{\frac{T}{2}+\delta} \int_{\alpha+\eta}^{\beta-\eta} f(x, t) d x d t \leq C \int_{0}^{T} \int_{\alpha}^{\beta} f(x, t) d x d t, \\
& \int_{\frac{T}{2}-\delta}^{\frac{T}{2}+\delta} \int_{0}^{\alpha+\eta} f(x, t) d x d t \leq C \int_{0}^{T} \int_{\alpha}^{\beta} f(x, t) d x d t .
\end{aligned}
$$

Proof It is obvious that (2.16) holds. Now we prove (2.15). We use a technique from Haraux [13] and consider $x$ as the evolution variable.

Set $\delta=\frac{1}{4}\left(T-T_{0}\right), \eta=\min \left\{\frac{\beta-\alpha}{4}, \frac{\delta}{4}\right\}$ and $R=\|g(a)\|_{\infty}$.

For all $x \in[0,1]$ and $\xi \in \mathbb{R}$ such that $0 \leq x-\xi \leq \frac{T}{2}$, we have

$$
\begin{aligned}
& \frac{d}{d x} \int_{x-\xi}^{T-(x-\xi)} f(x, t) d t \\
= & -f(x, T-(x-\xi))-f(x, x-\xi)+\int_{x-\xi}^{T-(x-\xi)}\left(2 P_{t} P_{t x}+2 P_{x} P_{x x}\right) d t \\
= & -f(x, T-(x-\xi))-f(x, x-\xi)+\left.2 P_{t} P_{x}\right|_{t=x-\xi} ^{t=T-(x-\xi)}+\int_{x-\xi}^{T-(x-\xi)} 2\left(P_{x} P_{x x}-P_{x} P_{t t}\right) d t \\
= & -\left(P_{t}(x, T-(x-\xi))-P_{x}(x, T-(x-\xi))\right)^{2} \\
& \quad-\left(P_{t}(x, x-\xi)+P_{x}(x, x-\xi)\right)^{2}-\int_{x-\xi}^{T-(x-\xi)} 2 g(a) P_{t} P_{x} d t \\
\leq & R \int_{x-\xi}^{T-(x-\xi)}\left(P_{t}^{2}+P_{x}^{2}\right) d t=R \int_{x-\xi}^{T-(x-\xi)} f(x, t) d t .
\end{aligned}
$$

Combining the above inequality with Gronwall's inequality, for $\xi \leq x_{0} \leq x$, we have

$$
\int_{x-\xi}^{T-(x-\xi)} f(x, t) d t \leq e^{R\left(x-x_{0}\right)} \int_{x_{0}-\xi}^{T-\left(x_{0}-\xi\right)} f\left(x_{0}, t\right) d t .
$$


Let $x \in(\beta-\eta, 1), \xi \in(\beta-4 \eta, \beta-2 \eta)$. Then (2.18) holds for $x_{0}=\xi+\eta$, that is

$$
\int_{x-\xi}^{T-(x-\xi)} f(x, t) d t \leq e^{R(x-\xi-\eta)} \int_{\eta}^{T-\eta} f(\xi+\eta, t) d t .
$$

Noticing

$$
x-\xi \leq 1-(\beta-4 \eta) \leq 1-\beta+\delta \leq \frac{T_{0}}{2}+\delta=\frac{T}{2}-\delta,
$$

and

$$
T-(x-\xi) \geq T-\left(\frac{T}{2}-\delta\right)=\frac{T}{2}+\delta,
$$

we have from $(2.19)$

$$
\int_{\frac{T}{2}-\delta}^{\frac{T}{2}+\delta} f(x, t) d t \leq e^{R(x-\xi-\eta)} \int_{\eta}^{T-\eta} f(\xi+\eta, t) d t .
$$

From the average formula for integrals it follows that there exists some $\sigma_{0} \in(\beta-3 \eta, \beta-2 \eta)$ such that

$$
\frac{1}{\eta} \int_{\beta-3 \eta}^{\beta-2 \eta} f(\sigma, t) d \sigma=f\left(\sigma_{0}, t\right) .
$$

Let $\xi_{0}=\sigma_{0}-\eta$. Then $\xi_{0} \in(\beta-4 \eta, \beta-2 \eta)$ and (2.20) can be applied to $\xi=\xi_{0}$, that is

$$
\int_{\frac{T}{2}-\delta}^{\frac{T}{2}+\delta} f(x, t) d t \leq e^{R\left(x-\sigma_{0}\right)} \int_{\eta}^{T-\eta} f\left(\sigma_{0}, t\right) d t .
$$

Combining (2.22) with (2.21) and noticing that $\sigma_{0}>\beta-3 \eta$, we have for all $x \in(\beta-\eta, 1)$

$$
\int_{\frac{T}{2}-\delta}^{\frac{T}{2}+\delta} f(x, t) d t \leq \frac{1}{\eta} e^{R(x-\beta+3 \eta)} \int_{\eta}^{T-\eta} \int_{\beta-3 \eta}^{\beta-2 \eta} f(\sigma, t) d \sigma d t .
$$

Integrating (2.23) with respect to those $x \in(\beta-\eta, 1)$ and noticing that $(\beta-3 \eta, \beta-2 \eta) \subset(\alpha, \beta)$, (2.15) immediately holds.

One can prove (2.17) using the same method.

Now we are in the position to prove Theorem 2.1.

Proof of Theorem 2.1 From Lemma 2.4, we have

$$
\int_{\frac{T}{2}-\delta}^{\frac{T}{2}+\delta} \int_{0}^{1}\left(P_{t}^{2}+P_{x}^{2}\right) d x d t \leq C \int_{0}^{T} \int_{\alpha}^{\beta}\left(P_{t}^{2}+P_{x}^{2}\right) d x d t .
$$

On the other hand, it is easy to find from (2.11) that

$$
\frac{d}{d t} \int_{0}^{1}\left(P_{t}^{2}(t, x)+P_{x}^{2}(t, x)\right) d x=2 \int_{0}^{1} g(a) P_{t}^{2}(t, x) d x \leq 2 R \int_{0}^{1}\left(P_{t}^{2}(t, x)+P_{x}^{2}(t, x)\right) d x .
$$

Combining the above inequality with Gronwall's inequality, we have for $0 \leq t \leq T$

$$
\int_{0}^{1}\left(P_{t}^{2}(T, x)+P_{x}^{2}(T, x)\right) d x \leq e^{2 R(T-t)} \int_{0}^{1}\left(P_{t}^{2}(t, x)+P_{x}^{2}(t, x)\right) d x .
$$

Combining (2.26) with (2.24), we have

$$
\left\|\left(P^{T}, Q^{T}\right)^{t r}\right\|_{H}^{2}=\frac{1}{2 \delta} \int_{\frac{T}{2}-\delta}^{\frac{T}{2}+\delta} \int_{0}^{1}\left(P_{t}^{2}(T, x)+P_{x}^{2}(T, x)\right) d x d t \leq \frac{C e^{2 R T}}{2 \delta} \int_{0}^{T} \int_{\alpha}^{\beta}\left(P_{t}^{2}+P_{x}^{2}\right) d x d t .
$$


Thanks to the translation invariance of the hyperbolic system, we know that from the inequality above for every $\varepsilon>0$ with $T-2 \varepsilon>T_{0}$, one has

$$
\left\|\left(P^{T}, Q^{T}\right)^{t r}\right\|_{H}^{2} \leq \frac{C e^{2 R T}}{2 \delta} \int_{\varepsilon}^{T-\varepsilon} \int_{\alpha}^{\beta}\left(P_{t}^{2}+P_{x}^{2}\right) d x d t \leq \frac{C e^{2 R T}}{2 \delta} \int_{0}^{T} \int_{\alpha}^{\beta}\left(r^{2} P_{t}^{2}+r^{2} P_{x}^{2}\right) d x d t,
$$

where $r \in C_{0}^{\infty}([0, T])$ with $0 \leq r \leq 1$ and $r \equiv 1$ on $[\varepsilon, T-\varepsilon]$.

Since $\left(P, P_{t}\right)^{t r} \in C([0, T] ; H)$, we have $P \in L^{2}\left(\omega ; H^{1}(0, T)\right)$ and $r P \in L^{2}\left(\omega ; H_{0}^{1}(0, T)\right)$. On the other hand, by the equation $P_{x x}=P_{t t}-g(a) P_{t}$, we deduce that $P_{x x} \in L^{2}\left(\omega ; H^{-1}(0, T)\right)$ and

$$
\left\|P_{x x}\right\|_{L^{2}\left(\omega ; H^{-1}(0, T)\right)} \leq C(1+R)\left\|P_{t}\right\|_{L^{2}\left(\omega ; L^{2}(0, T)\right)} .
$$

It is easy to find that $r P_{x x} \in L^{2}\left(\omega ; H^{-1}(0, T)\right)$ and

$$
\left\|r P_{x x}\right\|_{L^{2}\left(\omega ; H^{-1}(0, T)\right)} \leq C(1+R)\left\|P_{t}\right\|_{L^{2}\left(\omega ; L^{2}(0, T)\right)} .
$$

To complete the proof, we employ the interpolation theory (see [16]). From the proof of Theorem 12.3 of [16], we have $\left[H_{0}^{1}(0, T), H^{-1}(0, T)\right]_{\frac{1}{2}}=L^{2}(0, T)$. Thanks to Proposition 2.2 of [16], one has $r P_{x} \in L^{2}\left(\omega ; L^{2}(0, T)\right)$. Noticing that $r \in C_{0}^{\infty}([0, T])$ with $0 \leq r \leq 1$ and $r \equiv 1$ on $[\varepsilon, T-\varepsilon]$, we have from (2.25) and the fact that $g$ is nonnegative

$$
\left\|r^{\prime} P\right\|_{L^{2}\left(\omega ; L^{2}(0, T)\right)}^{2}=\left(\int_{0}^{\varepsilon}+\int_{T-\varepsilon}^{T}\right) \int_{\omega}\left(r^{\prime} P\right)^{2} d x d t \leq\left(\int_{0}^{\varepsilon}+\int_{T-\varepsilon}^{T}\right) \int_{\Omega}\left(r^{\prime} P\right)^{2} d x d t \leq C \varepsilon\left\|\left(P^{T}, Q^{T}\right)^{t r}\right\|_{H}^{2} .
$$

From Theorem 2.3 (intermediate derivatives theorem) of [16] and the two inequalities above, we have

$$
\begin{aligned}
\left\|r P_{x}\right\|_{L^{2}\left(\omega ; L^{2}(0, T)\right)}^{2} & \leq C\left(\int_{\omega}\left(\|r P\|_{H_{0}^{1}(0, T)}^{2}+\left\|r P_{x x}\right\|_{H^{-1}(0, T)}^{2}\right) d x\right) \\
& \leq C\left(\left\|r^{\prime} P\right\|_{L^{2}\left(\omega ; L^{2}(0, T)\right)}^{2}+\left\|r P_{t}\right\|_{L^{2}\left(\omega ; L^{2}(0, T)\right)}^{2}+\left\|r P_{x x}\right\|_{L^{2}\left(\omega ; H^{-1}(0, T)\right)}^{2}\right) \\
& \leq C \varepsilon\left\|\left(P^{T}, Q^{T}\right)^{t r}\right\|_{H}^{2}+C(1+R)\left\|P_{t}\right\|_{L^{2}\left(\omega ; L^{2}(0, T)\right)}^{2}
\end{aligned}
$$

for every $\varepsilon>0$. Now combining (2.27) and (2.28), taking $\varepsilon$ small enough, we complete the proof of Theorem 2.1.

\subsection{Fixed-point arguments}

In this subsection we prove Theorem 1.1 using Schaefer's fixed-point theorem (see Theorem 9.2.4 of $[10])$.

From Theorem 2.1, Lemma 2.1 and Lemma 2.2, we know that for every $a \in L^{\infty}((0, T) \times \Omega)$ and $\left(z^{0}, z^{1}\right) \in H$, there exists a control $h \in L^{2}((0, T) \times \omega)$ such that the system

$$
\left\{\begin{array}{l}
y_{t t}-y_{x x}+g(a) y_{t}=h 1_{\omega}, \quad \text { in }(0, T) \times \Omega, \\
y(t, 0)=y(t, 1)=0, \quad \text { in }(0, T), \\
y(0, x)=y_{t}(0, x)=0, \quad \text { in } \Omega, \\
y(T, x)=z^{0}(x), \quad y_{t}(T, x)=z^{1}(x), \quad \text { in } \Omega
\end{array}\right.
$$


admits a unique solution $y \in C\left([0, T] ; H_{0}^{1}(\Omega)\right) \cap C^{1}\left([0, T] ; L^{2}(\Omega)\right)$.

Therefore we can define a map

$$
K: L^{\infty}((0, T) \times \Omega) \rightarrow L^{\infty}((0, T) \times \Omega)
$$

by $K(a)=y$ where $y$ is the solution of $(2.29)$ with a suitable control $h \in L^{2}((0, T) \times \omega)$. To complete the proof of Theorem 1.1, it is sufficient to show $K$ has a fixed point.

First we have the following lemma.

Lemma 2.5 Let $T>0, M>0$. Then there exists a constant $C^{\prime}=C^{\prime}(T, \Omega, M)>0$ such that, for every $a \in L^{\infty}((0, T) \times \Omega)$ satisfying $\|a\|_{\infty} \leq M,\left(z^{0}, z^{1}\right) \in H$ and $h \in L^{2}((0, T) \times \omega)$, the solution of the system (2.29) satisfies

$$
\|y\|_{L^{\infty}\left((0, T) ; H_{0}^{1}(\Omega)\right)}^{2}+\left\|y_{t}\right\|_{L^{\infty}\left((0, T) ; L^{2}(\Omega)\right)}^{2} \leq C^{\prime}\left\|\left(z^{0}, z^{1}\right)\right\|_{H}^{2} .
$$

Proof Define $E(t)=\int_{\Omega}\left(y_{t}^{2}(t, x)+y_{x}^{2}(t, x)\right) d x$. Noticing that $g(a) \geq 0$, it is easy to have

$$
\frac{d}{d t} E(t)=-2 \int_{\omega} g(a) y_{t}^{2} d x+2 \int_{\omega} h 1_{\omega} y_{t} d x \leq E(t)+\int_{\omega} h^{2} d x
$$

So we have by Gronwall's inequality

$$
E(t) \leq C \int_{0}^{T} \int_{\omega} h^{2} d x d t, \quad \forall t \in[0, T] .
$$

On the other hand, from Lemma 2.2 and Theorem 2.1, we have the control $h$ satisfies

$$
\int_{0}^{T} \int_{\omega} h^{2} d x d t \leq\left(C_{1} e^{C_{2}\|g(a)\|_{\infty}}\right)^{2}\left\|\left(z^{0}, z^{1}\right)\right\|_{H}^{2}
$$

Then from (2.31), (2.32) and the continuity of $g$, we get (2.30) which completes the proof of Lemma 2.5 .

Proof of Theorem 1.1 Lemma 2.5 shows that $K$ maps bounded sets of $L^{\infty}((0, T) \times \Omega)$ into bounded sets of $C\left([0, T] ; H_{0}^{1}(\Omega)\right) \cap C^{1}\left([0, T] ; L^{2}(\Omega)\right)$. Moreover, by the compactness of embedding

$$
C\left([0, T] ; H_{0}^{1}(\Omega)\right) \cap C^{1}\left([0, T] ; L^{2}(\Omega)\right) \rightarrow L^{\infty}((0, T) \times \Omega),
$$

we know that the map $K$ is compact and continuous.

From Schaefer's fixed point theorem, in order to prove $K$ has fixed points, it is sufficient to show the set

$$
S=\left\{y \in L^{\infty}((0, T) \times \Omega): y=\lambda K y \text { for some } 0 \leq \lambda \leq 1\right\}
$$

is bounded.

By the definition of $K, y \in S$ solves the following problem :

$$
\left\{\begin{array}{l}
y_{t t}-y_{x x}+g(\lambda y) y_{t}=h 1_{\omega}, \quad \text { in }(0, T) \times \Omega \\
y(t, 0)=y(t, 1)=0, \quad \text { in }(0, T) \\
y(0, x)=y_{t}(0, x)=0, \quad \text { in } \Omega \\
y(T, x)=z^{0}(x), \quad y_{t}(T, x)=z^{1}(x), \quad \text { in } \Omega .
\end{array}\right.
$$


From the proof of Lemma 2.5, we have the solution $y$ of (2.33) satisfies

$$
\|y\|_{\infty} \leq C C_{1} e^{C_{2}\|g(\lambda y)\|_{\infty}}\left\|\left(z^{0}, z^{1}\right)\right\|_{H} .
$$

From the condition (1.4) we know that there exists $b>0$ such that

$$
\|g(\lambda y)\|_{\infty} \leq \gamma \ln \left(\|\lambda y\|_{\infty}\right)+b, \quad \forall\|y\|_{\infty} \geq 1 .
$$

Substituting (2.35) into (2.34), we have

$$
\|y\|_{\infty} \leq 1+C C_{1} e^{C_{2} b} e^{C_{2} \gamma \ln \|y\|_{\infty}} .
$$

Taking $\gamma>0$ small enough such that $C_{2} \gamma \leq \frac{1}{2}$, then we get

$$
\sqrt{\|y\|_{\infty}} \leq C C_{1}^{2} e^{2 C_{2} b}
$$

from which we deduce that $S$ is bounded. Thus we complete the proof of Theorem 1.1.

\section{Proof of Theorem 1.2}

In this section, we consider the controllability of the system

$$
\left\{\begin{array}{l}
y_{t t}-y_{x x}+|y| y_{t}=h 1_{\omega} \quad \text { in }(0, T) \times \Omega \\
y(t, 0)=y(t, 1)=0 \quad \text { in }(0, T)
\end{array}\right.
$$

Set $T>T_{0}$. We shall prove that there exists $r_{0}>0$ such that for every $\left(y^{0}, y^{1}\right),\left(z^{0}, z^{1}\right) \in H$, with $\left\|\left(y^{0}, y^{1}\right)\right\|_{H} \leq r_{0}$ and $\left\|\left(z^{0}, z^{1}\right)\right\|_{H} \leq r_{0}$, there exists $h \in L^{2}((0, T) \times \omega)$ such that the solution of (3.1) satisfies

$$
y(0, x)=y^{0}(x), \quad y_{t}(0, x)=y^{1}(x), \quad x \in \Omega
$$

and

$$
y(T, x)=z^{0}(x), \quad y_{t}(T, x)=z^{1}(x), \quad x \in \Omega .
$$

Proof of Theorem 1.2 Step 1. Let us first prove that for every $\xi \in B$, there exists $h \in L^{2}((0, T) \times$ $\omega)$ such that the solution of the linearized system of (3.1)

$$
\left\{\begin{array}{l}
z_{t t}-z_{x x}+|\xi| \xi_{t}=h 1_{\omega} \quad \text { in }(0, T) \times \Omega \\
z(t, 0)=z(t, 1)=0 \quad \text { in }(0, T)
\end{array}\right.
$$

satisfies

$$
z(0, x)=y^{0}(x), \quad z_{t}(0, x)=y^{1}(x), \quad z(T, x)=z^{0}(x), \quad z_{t}(T, x)=z^{1}(x), \quad x \in \Omega .
$$

Consider the system

$$
\begin{cases}y_{1, t t}-y_{1, x x}+f=0 & \text { in }(0, T) \times \Omega \\ y_{1}(t, 0)=y_{1}(t, 1)=0 & \text { in }(0, T) \\ y_{1}(0, x)=y_{1, t}(0, x)=0 & \text { in } \Omega .\end{cases}
$$


From classic theories on the existence of solutions for PDEs (see, for example, [18] or Theorem 2.2 of [15]), it is known that for any $f \in L^{1}\left(0, T ; L^{2}(\Omega)\right)$, system (3.4) admits a unique solution $y_{1} \in B$ such that $\left\|y_{1}\right\|_{B} \leq C\|f\|_{L^{1}\left(0, T ; L^{2}(\Omega)\right)}$. Let $\Psi$ be the continuous linear map

$$
\begin{array}{cc}
L^{1}\left(0, T ; L^{2}(\Omega)\right) & \rightarrow B \\
f & \mapsto y_{1},
\end{array}
$$

where $y_{1}$ is the solution of (3.4) with $f \in L^{1}\left(0, T ; L^{2}(\Omega)\right)$.

Given $\left(y^{0}, y^{1}\right) \in H$, let $y_{2} \in B$ be the solution of

$$
\left\{\begin{array}{l}
y_{2, t t}-y_{2, x x}=0 \quad \text { in }(0, T) \times \Omega \\
y_{2}(t, 0)=y_{2}(t, 1)=0 \quad \text { in }(0, T) \\
y_{2}(0, x)=y^{0}(x), \quad y_{2, t}(0, x)=y^{1}(x), \quad \text { in } \Omega .
\end{array}\right.
$$

Let $S$ be the continuous linear map

Consider the control problem

$$
\begin{array}{ccc}
H & \rightarrow & B \\
\left(y^{0}, y^{1}\right) & \mapsto & y_{2} .
\end{array}
$$

$$
\left\{\begin{array}{l}
y_{3, t t}-y_{3, x x}=h 1_{\omega} \quad \text { in }(0, T) \times \Omega \\
y_{3}(t, 0)=y_{3}(t, 1)=0 \quad \text { in }(0, T),
\end{array}\right.
$$

where $h$ is the internal control. Since system (3.6) is exactly controllable in $H$ for $T>T_{0}$ (see [2] and [13]), there exists $h \in L^{2}((0, T) \times \omega)$ such that the solution of $(3.6)$ satisfies

$$
\begin{gathered}
y_{3}(0, x)=y_{3, t}(0, x)=0, \quad x \in \Omega, \\
y_{3}(T, x)=z^{0}(x)-y_{1}(T, x)-y_{2}(T, x), \quad x \in \Omega,
\end{gathered}
$$

and

$$
y_{3, t}(T, x)=z^{1}(x)-y_{1, t}(T, x)-y_{2, t}(T, x), \quad x \in \Omega .
$$

Let $\Gamma$ be the continuous linear map

$$
\begin{array}{cl}
L^{2}((0, T) \times \omega) & \rightarrow B \\
h & \mapsto y_{3},
\end{array}
$$

where $y_{3}$ is the solution of (3.6) with (3.7), (3.8) and (3.9).

Having defined $\Psi, S$ and $\Gamma$ above, by an easy computation one finds that $\Psi\left(|\xi| \xi_{t}\right)+S\left(y^{0}, y^{1}\right)+\Gamma h$ solves (3.2) with (3.3).

Step 2. We will complete the proof of Theorem 2.1 by constructing a contractive map.

As it was shown in [31], among the infinite controls $h$ such that the solution of (3.6) satisfies (3.7), (3.8) and (3.9), there exists $\hat{\varphi} \in L^{2}((0, T) \times \omega)$ which has the minimal $L^{2}$-norm. Also $\hat{\varphi}$ solves

$$
\left\{\begin{array}{l}
\varphi_{t t}-\varphi_{x x}=0 \quad \text { in }(0, T) \times \Omega \\
\varphi(t, 0)=\varphi(t, 1)=0 \quad \text { in }(0, T) \\
\varphi(0, x)=\hat{\varphi}^{0}(x), \quad \varphi_{t}(0, x)=\hat{\varphi}^{1}(x), \quad \text { in } \Omega
\end{array}\right.
$$


where $\left(\hat{\varphi}^{0}, \hat{\varphi}^{1}\right) \in L^{2}(\Omega) \times H^{-1}(\Omega)$ is the minimizer of the following functional

$$
J\left(\varphi^{0}, \varphi^{1}\right)=\frac{1}{2} \int_{0}^{T} \int_{\omega} \varphi^{2} d x d t-\left(\varphi(T), y_{3, t}(T)\right)_{L^{2}}+\left\langle\varphi_{t}(T), y_{3}(T)\right\rangle_{H^{-1}, H_{0}^{1}}
$$

And the following identity holds :

$$
\int_{0}^{T} \int_{\omega} \hat{\varphi}^{2} d x d t=\left(\hat{\varphi}(T), y_{3, t}(T)\right)_{L^{2}}-\left\langle\hat{\varphi}_{t}(T), y_{3}(T)\right\rangle_{H^{-1}, H_{0}^{1}}
$$

Since $T>T_{0}$, the following observability inequality holds

$$
\|\hat{\varphi}(T)\|_{L^{2}(\Omega)}^{2}+\left\|\hat{\varphi}_{t}(T)\right\|_{H^{-1}(\Omega)}^{2} \leq C \int_{0}^{T} \int_{\omega} \hat{\varphi}^{2} d x d t .
$$

So from (3.12) and (3.13) we have

$$
\left.\int_{0}^{T} \int_{\omega} \hat{\varphi}^{2} d x d t \leq C\left(\left\|y_{3}(T, \cdot)\right\|_{H_{0}^{1}(\Omega)}^{2}+\| y_{3, t}(T, \cdot)\right) \|_{L^{2}(\Omega)}^{2}\right) .
$$

Now define the map $F: B \rightarrow B$ by

$$
F \xi=\Psi\left(|\xi| \xi_{t}\right)+S\left(y^{0}, y^{1}\right)+\Gamma \hat{\varphi}
$$

Then to get exact controllability of system (3.1) it is sufficient to prove that $F$ has a fixed point .

Using the continuity of $\Psi, S$ and $\Gamma$, from (3.8), (3.9), (3.14) and (3.15), it is not difficult to check that there exists $K>0$ which is independent of $\xi,\left(y^{0}, y^{1}\right)$ and $\left(z^{0}, z^{1}\right)$ such that

$$
\|F \xi\|_{B}=\left\|\Psi\left(|\xi| \xi_{t}\right)+S\left(y^{0}, y^{1}\right)+\Gamma \hat{\varphi}\right\|_{B} \leq K\left(\|\xi\|_{B}^{2}+\left\|\left(y^{0}, y^{1}\right)\right\|_{H}+\left\|\left(z^{0}, z^{1}\right)\right\|_{H}\right)
$$

and

$$
\|F \xi-F \zeta\|_{B} \leq K\|\xi-\zeta\|_{B}\left(\|\xi\|_{B}+\|\zeta\|_{B}\right)
$$

Take

$$
R=\frac{1}{4 K}, \quad r_{0}=\frac{3 R^{2}}{2}
$$

Let

$$
\left\|\left(y^{0}, y^{1}\right)\right\|_{H} \leq r_{0} \text { and }\left\|\left(z^{0}, z^{1}\right)\right\|_{H} \leq r_{0} .
$$

Set

$$
B_{R}=\left\{\xi \in B:\|\xi\|_{B} \leq R\right\},
$$

which is a closed subsets of the Banach space $B$. Then we have

$$
F\left(B_{R}\right) \subset B_{R}
$$

and

$$
\|F \xi-F \zeta\| \leq \frac{1}{2}\|\xi-\zeta\|_{B}, \quad \forall \xi, \zeta \in B_{R} .
$$

Hence, by the Banach fixed-point theorem $F$ has a unique fixed point in $B_{R}$. 
Remark 3.1 We only consider the case $g(s)=|s|$ in Theorem 1.2 for simplicity. In fact, using the same method, the result of Theorem 1.2 can be easily extended to the case that $g(s)$ is locally Lipschitz continuous around zero. Thus one can also prove that system(1.1) with $g(s)=|s|^{m}(m>1)$ is locally controllable. It seems to be much more difficult or impossible to prove that the map $F$ constructed in the proof of Theorem 1.2 is contractive for the case that $g(s)=|s|^{m}$ with $0<m<1$ since now $g(s)$ is not Lipschtiz continuous. However, we guess that the local controllability may be still true for this case. But to prove this, some new methods must be exploited.

Acknowledgments The authors would like to express their sincere thanks to the referees for their valuable comments.

\section{References}

[1] K. Agre and M.A.Rammaha, Global solutions to boundary value problems for a nonlinear wave equation in high space dimensions, Differential Integral Equations, 14 (2001), 1315-1331.

[2] C. Bardos, G. Lebeau, and J. Rauch, Sharp sufficient conditions for the observation, control and stabilization of waves from the boundary, SIAM J. Control Optim., 30 (1992) 1024-1065.

[3] V. Barbu, Nonlinear Differential Equations in Banach Spaces, Nordhoff, 1976.

[4] V. Barbu, I. Lasiecka, and M. A. Rammaha, On nonlinear wave equations with degenerate damping and source terms, Trans. Amer. Math. Soc., 357 (7) (2005) 2571-2611.

[5] A. Carrasco, H. Leiva and J. Sanchez, Controllability of the semilinear beam equation. J. Dyn. Control Syst., 19(4) (2013) 553-568.

[6] F. W. Chaves-Silva, L. Rosier and E. Zuazua, Null controllability of a system of viscoelasticity with a moving control. J. Math. Pures Appl., 101 (2014) 198-222.

[7] J. M. Coron, Control and Nonlinearity, Mathematical Surveys and Monographs, 136, AMS, Providence, RI, 2007.

[8] Dang Dinh Ang, A. Pham Ngoc Dinh, Mixed problems for some semi-linear wave equation with a nonhomogeneous condition, Nonlinear Anal., 12(1988), 581-592.

[9] T. Duyckaerts, X. Zhang and E. Zuazua, On the optimality of the observability inequalities for parabolic and hyperbolic systems with potentials, Ann. Inst. H. Poincaré Anal. Non Linéaire, 25 (2008), $1-41$.

[10] L. C. Evans, Partial Differential Equations, Graduate Studies in Mathematics, vol. 19, AMS, RI, 1998. 
[11] X. Fu, J. Yong and X. Zhang, Exact controllability for the multidimensional semilinear hyperbolic equations, SIAM J. Control Optim., 46 (2007), 1578-1614.

[12] A. Haraux, Nonlinear Evolution Equations-Global Behaviour of Solutions, Lecture Notes in Mathematics, 841, Springer-Verlag, 1981.

[13] A. Haraux, A generalized internal control for the wave equation in a rectangle, J. Math. Anal. Appl., 153 (1990) 190-216.

[14] K. Jörgens, Das Anfangswertproblem im Grossen für eine Klasse nichtlinearer Wellengleichungen, Math. Z., 77 (1961) 295-308.

[15] I. Lasiecka, J. L. Lions and R. Triggiani, Nonhomogeneous boundary value problems for second order hyperbolic operators, J. Math. Pures Appl., 69 (1986) 149-192.

[16] J. L. Lions, E. Magenes, Non-Homogeneous Boundary Value Problems and Applications I, Springer-Verlag, New York-Heidelberg-Berlin, 1972.

[17] P. Martin, L. Rosier and P. Rouchon, Null controllability of the structurally damped wave equation with moving control. SIAM J. Control Optim., 51(1) (2013) 660-684.

[18] A. Pazy, Semigroups of Linear Operators and Applications to Partial Differential Equations, Applied Mathematical Sciences, vol. 44, Speinger-Verlag, New York, 1983.

[19] M. A. Rammaha and T. A. Strei, Global existence and nonexistence for nonlinear wave equations with damping and source terms, Trans. Amer. Math. Soc., 354 (9) (2002) 3621-3637.

[20] I. E. Segal, Non-linear Semigroups, Annals of Math., 78 (1963) 339-364.

[21] M. A. Shubov, C. F. Martin, J. P. Dauer and B. P. Belinskiy, Exact controllability of the damped wave equation. SIAM J. Control Optim., 35(5) (1997) 1773-1789.

[22] L. Tebou, Some results on the controllability of coupled semilinear wave equations: the desensitizing control case. SIAM J. Control Optim., 49(3) (2011) 1221-1238.

[23] J. Vancostenoble, Exact controllability of a damped wave equation with distributed controls, Acta Math. Hungar., 89 (1-2) (2000) 71-92.

[24] J. Wu, X, Zhu and S. Li, Simultaneous controllability of damped wave equations,submitted.

[25] P. F. Yao, Modeling, Analysis and Control in Structural Dynamics: with a differential geometric approach, Chapman and Hall/CRC Applied Mathematics and Nonlinear Science, Taylor and Francis, 2011. 
[26] X. Zhang, Explict observability inequalities for the wave equation with lower order terms by means of Carleman inequalities, SIAM J. Control Optim., 39 (3) (2000) 812-834.

[27] X. Zhang, Remarks on the controllability of some quasilinear equations, http:// arxiv.org /abs/0904.2427vl.

[28] Y. Zhou, Z. Lei, Local exact boundary controllability for nonlinear wave equations, SIAM J. Control Optim., 46 (2007), 1022-1051.

[29] K. Zhuang, T. Li and B. Rao, Exact controllability for first quasilinear hyperbolic systems with internal control, Discrete and Continuous Dynamical System, 36 (2) (2016), 1105-1124.

[30] E. Zuazua, Exact controllability for semilinear wave equations in one space dimension, Ann. Inst. H. Poincaré Analyse Non Linéare, 10 (1993) 109-129.

[31] E. Zuazua, Controllability and Observability of Partial Differential Equations: Some results and open problems, Handbook of Differential Equations: Evolutionary Differential Equations, vol. 3, Elsevier Science, 2006, 527-621. 